\title{
Exploring the Spectrum of Regularized Bosonic String Theory ${ }^{1}$
}

\author{
J. Ambjørn ${ }^{a, b *}$ and Y. Makeenko ${ }^{a, c * * *}$ \\ ${ }^{a}$ The Niels Bohr Institute, Copenhagen University, Copenhagen, DK-2100 Denmark \\ ${ }^{b}$ IMAPP, Radboud University, Nijmegen, 6525, AJ, The Netherlands \\ ${ }^{c}$ Institute of Theoretical and Experimental Physics, Moscow, 117218 Russia \\ *e-mail:ambjorn@nbi.dk \\ **e-mail:makeenko@nbi.dk \\ Received October 20, 2014
}

\begin{abstract}
We implement a UV regularization of the bosonic string by truncating its mode expansion and keeping the regularized theory "as diffeomorphism invariant as possible." We compute the regularized determinant of the $2 \mathrm{~d}$ Laplacian for the closed string winding around a compact dimension, obtaining the effective action in this way. The minimization of the effective action reliably determines the energy of the string ground state for a long string and/or for a large number of space-time dimensions. We discuss the possibility of a scaling limit when the cutoff is taken to infinity.
\end{abstract}

Contribution for the JETP special issue in honor of V.A.Rubakov's 60th birthday

DOI: $10.1134 / \mathrm{S} 1063776115030024$

\section{INTRODUCTION}

A modern formulation of string theory is based on the Polyakov path integral [1], where the worldsheet metric $g_{a b}(\omega)$ and the target-space position $X^{\mu}(\omega), \mu=$ $1, \ldots, d$, of the string worldsheet are treated as independent variables. Thanks to the diffeomorphism invariance, the metric can be diagonalized, $g_{a b}=e^{\varphi} \delta_{a b}$, by choosing the conformal gauge. The remaining path integration over the so-called Liouville field $\varphi$ decouples on the mass shell for the bosonic string in $d=26$, the critical dimension. Due to this decoupling, the results in $d=26$ reproduce those obtained in the early 1970 s using the operator formalism. For $d \neq$ 26 , the path integral over $\varphi$ has to be taken into account and plays an important role for the consistency of the theory.

The path integral over the target-space string coordinates (and ghosts) is Gaussian and results in a determinant of the $2 \mathrm{~d}$ Laplace-Beltrami operator with proper boundary conditions imposed. For an open string with fixed ends, these are Dirichlet boundary conditions, for which the determinant was computed in $[2,3]$. The result is given by the conformal anomaly and determines the effective action for the Liouville field $\varphi$. The path integral over $\varphi$ can be consistently treated [4] order by order in the inverse string length and/or in the limit of a large number of space-time dimensions $d$, where the WKB expansion around the saddle points applies. Of special interest in this

\footnotetext{
${ }^{1}$ The article is published in the original.
}

approach is the ground-state energy as a function of the string length $R$, which is given by the well-known Alvarez-Arvis spectrum $[5,6]$. It reveals a tachyonic singularity at distances $R \leq R_{0}$, with $-1 / R_{0}^{2}$ being the tachyon mass squared. For larger distances, this quantity is well-behaved.

The conformal factor does not appear in the classical string. However, as was pointed out by Polyakov [1], the computation of $2 \mathrm{~d}$ determinants requires a $\mathrm{UV}$ cutoff like $\Lambda^{2} \sqrt{g}$ in the parameter space. ${ }^{2}$ This follows from the diffeomorphism invariance and results in the conformal anomaly, which decouples in the effective action as $\Lambda \longrightarrow \infty$. The dependence of the cutoff on the metric is of crucial importance for the consideration in this paper.

The emergence of a tachyonic excitation of the string is seen clearly in the zeta-function regularization, where the sum over oscillators (the stringy modes) is computed as

$$
\sum_{n=1}^{\infty} n=\zeta(-1)=-\frac{1}{12} .
$$

This negative value is the result of an analytic continuation from positive values of the argument of the zeta function, for which the sum is convergent. Of course, the sum of positive numbers in Eq. (1) is infinite and the negative value emerges after the subtraction of an

\footnotetext{
${ }^{2}$ We recall that $\sqrt{g}=e^{\varphi}$ in the conformal gauge.
} 
infinity as was illustrated in detail by one of the first calculations [7]. In this paper, we investigate how the sums over the stringy modes (like in Eq. (1)) can be consistently regularized, maximally preserving the diffeomorphism invariance.

One regularization of this kind is the so-called dynamical triangulation (DT) [8], where the intrinsic geometry of the parameter space (defined by the metric $\left.g_{a b}(\omega)\right)$ is approximated by a set of equilateral triangles of side $a$. The summation over triangulations is done independently of the integration over the targetspace coordinates associated, for instance, with the vertices of the triangles. In this sense, DT discretizes the Polyakov string. DT provides the conceptual foundation for matrix-model solutions of the so-called non-critical string theory. However, for the real bosonic string theory with $d \geq 2$, DT also provides an interesting result. In DT, the renormalized mass excitations and the renormalized string tension are by definition non-negative and it was shown in [9] that if we keep the lowest mass excitation finite as the cutoff $a \longrightarrow 0$, the string tension scales to infinity. With this otherwise very successful regularization, it thus seems impossible to obtain a bosonic string with a finite tachyonic mass and a finite string tension.

In this paper, we want to make contact with the DT result mentioned using a standard continuum regularization of the bosonic string, namely, truncating the string mode expansion. We consider a closed string winding once around a compact dimension of length $R$ and propagating a (Euclidean) time $T$. We generically consider a string whose length is larger than the inverse tachyon mass (if present for the regularized string). We therefore expect a stable ground state and compute its mass as a function of the string length $R$. This determines the string tension as the energy per a unit length and should provide us with information about the mass of the lowest state (usually, the tachyon) from the behavior of the energy at small $R$. We then search for a scaling regime, where the two quantities may or may not remain finite in the limit of an infinite cutoff.

In Sections 2 and 3, we introduce the string regularization by a truncation of the mode expansion and compute the regularized determinant of the $2 \mathrm{~d}$ Laplacian for a $\omega_{T} \times \omega_{R}$ rectangle in the parameter space. We use the Dirichlet boundary condition along the $T$-axis and periodic boundary conditions along the $R$-axis. This gives an effective action of the regularized string. We demonstrate how the Lüscher term emerges using this regularization. In Section 4, we argue that the reparameterization invariance favors $N_{T}=N_{R} T / R$ for the numbers of modes $N_{T}$ and $N_{R}$ along the respective $T$ and $R$-axes.

The effective action computed this way depends on the ratio $\omega_{T} / \omega_{R}$. There are two cases where this parameter can be reliably determined by minimizing the effective action: small $\alpha^{\prime} / R^{2}$ and large $d$. They are considered in Sections 5 and 6. In Section 5, we first recall the situation in the classical limit and then analyze the one-loop (semiclassical) correction that determines the renormalization of the string tension. In Section 6, we derive the equation which minimizes the effective action at large $d$. The minimized effective action contains terms of all orders in $\alpha^{\prime} / R^{2}$, and we find the effective action in both the large- $R$ and the small- $R$ limit. We show that, at a finite cutoff, the tachyonic singularity is present for positive values of the bare string tension $K_{0}$, but is absent for a range of negative values of $K_{0}$. We find that there exists a critical (negative) value $K_{*}$ such that if the bare string tension $K_{0}$ approaches $K_{*}$ from above, it is possible to have a renormalized string tension $K$ that stays finite as the cutoff $\Lambda \longrightarrow \infty$, but in this case the lowest mass excitation does not scale but goes to infinity. However, there also exists a value $K_{c}, K_{*}<K_{c}<0$, such that if $K_{0}$ approaches $K_{c}$ from below, the lowest mass can be kept finite for the cutoff $\Lambda \longrightarrow \infty$, but in this case the "renormalized" string tension goes to infinity as $\Lambda^{2}$. This situation seems very similar to what is observed using DT as a regularization.

\section{REGULARIZED STRING MODE EXPANSION}

We consider a closed string winding one time around a compact dimension of length $R$. We impose Dirichlet boundary condition along the $T$-axis and periodic boundary condition along the $R$-axis. We consider an $\omega_{T} \times \omega_{R}$ rectangle in the parameter space mapped onto a $T \times R$ rectangle in the target space with 0 and $R$ identified along the $R$-axis.

The one-loop effective action can be computed as the determinant of the $2 \mathrm{~d}$ Laplacian in the conformal gauge with the above boundary conditions imposed on the $\omega_{T} \times \omega_{R}$ rectangle. The Laplacian is

$$
\Delta=\frac{1}{\rho}\left(\partial_{1}^{2}+\partial_{2}^{2}\right),
$$

where $\rho=R T / \omega_{R} \omega_{T}$, and we have

$$
\begin{gathered}
\operatorname{tr} \log \left(-\Delta a^{2}\right)=\sum_{m=1}^{\infty} \sum_{n=-\infty}^{\infty} \log \left\{\left[\left(\frac{\pi m}{\omega_{T}}\right)^{2}\right.\right. \\
\left.\left.+\left(\frac{2 \pi n}{\omega_{R}}\right)^{2}\right] \frac{\omega_{R} \omega_{T} a^{2}}{R T}\right\},
\end{gathered}
$$

where we want to think about $a=\pi / \Lambda$ as a UV latticelike cutoff similar to the lattice cutoff $a$ in DT. For large $\omega_{T} \gg \omega_{R}$, we replace the sum over $m$ by the integral over the "momentum" $x=\pi m / \omega_{T}$ :

$$
\begin{gathered}
\operatorname{tr} \log \left(-\Delta a^{2}\right) \\
=\frac{\omega_{T}}{\pi} \sum_{n} \int d x \log \left\{\left[x^{2}+\left(\frac{2 \pi n}{\omega_{R}}\right)^{2}\right] \frac{\omega_{R} \omega_{T} a^{2}}{R T}\right\} .
\end{gathered}
$$


To regularize this divergent expression, we integrate over $x$ from 0 to $X$, where the upper limit of the integration is introduced to provide a UV cutoff, which cuts off mode numbers $m$ larger than $X \omega_{T} / \pi$. In Section 4 , we relate it to the $\Lambda$ introduced above. To perform the integral, we use the relation

$$
\begin{gathered}
\int_{0}^{X} d x \log \left(x^{2}+y^{2}\right) \\
=X\left[\log \left(X^{2}+y^{2}\right)-2\right]+2 y \arctan \frac{X}{y},
\end{gathered}
$$

where $y=2 \pi n / \omega_{R}$. As $X \longrightarrow \infty$, in the right-hand side, we recover the term $\pi|y|$ familiar from the zeta-function regularization.

The remaining sum over $n$ can be evaluated by using Plana's summation formula

$$
\begin{gathered}
\frac{1}{2} f(0)+\sum_{n=1}^{\infty} f(n)=\int_{0}^{\infty} d \omega f(\omega) \\
+i \int_{0}^{\infty} d t \frac{f(i t)-f(-i t)}{e^{2 \pi t}-1},
\end{gathered}
$$

which holds whenever $f(z)$ is analytic for $\operatorname{Re} z \geq 0$.

The first term in the right-hand side of Eq. (6) results in the integral

$$
\begin{gathered}
\int_{0}^{X} d x \int_{-Y}^{Y} d y \log \left(x^{2}+y^{2}\right) \\
=\left(3 X^{2}+Y^{2}\right) \arctan \frac{Y}{X}+\left(X^{2}+3 Y^{2}\right) \arctan \frac{X}{Y} \\
+2 X Y\left[\log \left(X^{2}+Y^{2}\right)-3\right]-\frac{\pi}{2}\left(X^{2}+Y^{2}\right) .
\end{gathered}
$$

Here, $Y$ is a UV mode sum cutoff along the $\omega_{R}$ axis in the same way as $X$ was along the $\omega_{T}$ axis.

\section{LUSCHER TERM FROM THE MODE EXPANSION}

The second integral in the right-hand side of Eq. (6) is convergent and therefore does not depend on the cutoff $Y$. Substituting

$$
y \arctan \frac{X}{y} \stackrel{x \gg y>0}{\longrightarrow} \frac{\pi}{2} y
$$

and using

$$
\int_{0}^{\infty} d t \frac{t}{e^{2 \pi t}-t}=\frac{1}{24},
$$

we finally obtain

$$
\begin{array}{r}
\operatorname{tr} \log \left(-\Delta a^{2}\right)=\frac{\omega_{T} \omega_{R}}{2 \pi^{2}}\left\{\left(3 X^{2}+Y^{2}\right) \arctan \frac{Y}{X}\right. \\
+\left(X^{2}+3 Y^{2}\right) \arctan \frac{X}{Y} \\
+2 X Y\left[\log \left(\left(X^{2}+Y^{2}\right) \frac{\omega_{R} \omega_{T} a^{2}}{R T}\right)-3\right] \\
\left.-\frac{\pi}{2}\left(X^{2}+Y^{2}\right)\right\}-\frac{\pi \omega_{T}}{3 \omega_{R}}
\end{array}
$$

The last term in the right-hand side is nothing but the Lüscher term. It comes from the second term in the right-hand side of Eq. (6), that is, from the difference between the sum and the integral in the first term in the right-hand side of Eq. (6). The rest of the terms in (10) comes from the integral. This demonstrates how the Lüscher term emerges when we use truncation of the mode expansion as our regularization.

\section{THE CHOICE OF THE CUTOFF}

Equation (10) above is derived when the mode expansion is truncated at the number of modes $N_{T}=$ $\omega_{T} X / \pi$ and $N_{R}=\omega_{R} Y / \pi$, as one would do in a box. However, the box is here a "parameter spacetime box," and it becomes important to choose $X$ and $Y$ such that the reparameterization invariance be preserved as much as possible. Using the zeta-function regularization, we find that the ground-state energy of a given choice of $\omega_{T}$ and $\omega_{R}$ only depends on the ratio $\omega_{T} / \omega_{R}$.

In our case, the relation between $X, Y$, and the cutoff $\Lambda$ should be derived taking the boundary metric $T / \omega_{T}$ along the $T$-axis and $R / \omega_{R}$ along the $R$-axis into account. Following Polyakov's idea [1] to choose the cutoff for the chosen parameterization as $\Lambda \sqrt[4]{g}$, we arrive at

$$
X=\frac{T}{\omega_{T}} \Lambda, \quad Y=\frac{R}{\omega_{R}} \Lambda .
$$

It follows from these relations that the one-loop effective action depend only on the ratio $\omega_{T} / \omega_{R}$, like for the zeta-function regularization.

The DT regularization also favors relation (11). Using the DT regularization, we attach a random lattice with the lattice spacing $a$ at the boundary of the physical domain, i.e., the boundary with the length $T$ and $R$. Hence, physical wavelengths smaller than $a$ should not actually be allowed in the determinant calculation. When we calculate the determinant, we have $\delta x=\pi / k_{m}, k_{m}=m \pi / \omega_{T}$. Hence, $\delta x=\omega_{T} / m$. However, we have to scale the coordinate $x$ to a physical $x_{p h}=$ $\left(T / \omega_{T}\right) x$. Thus, $\delta x_{p h}>a$ results in $n<N_{T}=T / a$, i.e., $X=\left(T / \omega_{T}\right) \Lambda, \Lambda=\pi / a$. Similarly $Y=\left(R / \omega_{R}\right) \Lambda$ or $Y=$ $\left(R \omega_{T} / T \omega_{R}\right) X$. 


\section{SEMICLASSICAL EXPANSION IN $\alpha^{\prime}$}

Because $\alpha^{\prime} \propto \hbar$, the semiclassical WKB expansion, which is an expansion in $\hbar$, also becomes an expansion in $\alpha^{\prime}$. Since $\alpha^{\prime}$ has the dimension of length ${ }^{2}$, the actual expansion parameter is $\alpha^{\prime} / R^{2}$. Accordingly, the bare string tension is

$$
K_{0}=\frac{1}{2 \pi \alpha^{\prime}} .
$$

A renormalization of $K$ already occurs at the order $\hbar$. The lowest mass appears only at higher orders.

\subsection{Classical Limit}

By minimizing the quadratic action

$$
S_{0}=\frac{K_{0}}{2}\left(\frac{T^{2}}{\omega_{T}^{2}}+\frac{R^{2}}{\omega_{R}^{2}}\right) \omega_{R} \omega_{T},
$$

we obtain $T / \omega_{T}=R / \omega_{R}$ classically. This implies that we can choose

$$
\omega_{T}=T, \quad \omega_{R}=R .
$$

A possible constant factor between $\omega_{T}, \omega_{R}$ and $T, R$ does not affect the renormalized constants calculated below. Classically, we therefore have $X=Y=\Lambda$, but as we see below, this is not true when we include quantum corrections.

\subsection{Order $\hbar$}

If we use Eq. (14) in formula (10), we obtain the one-loop effective action

$$
S_{1}=\frac{d-2}{2 \pi^{2}} R T \Lambda^{2}\left[\log \left(2 \pi^{2}\right)+\frac{\pi}{2}-3\right]-\frac{\pi(d-2)}{6} \frac{T}{R} \text {. }
$$

In principle, we have to use generic corrections of the order $\alpha^{\prime}$ to Eq. (14) when substituting $\omega_{T}$ and $\omega_{R}$ into classical action (13). Therefore, we write

$$
\omega_{T}=T, \quad \omega_{R}=R+\frac{a_{1}}{K_{0} R} .
$$

However, this additional term cancels when substituted in Eq. (13), and is important only at the next order, where $a_{1}$ is determined by the minimization of the action.

Adding (13) and (15), we obtain the finite result

$$
S_{0}+S_{1}=K R T-\frac{\pi(d-2)}{6} \frac{T}{R},
$$

if we use the additive renormalization of the string tension

$$
K=K_{0}-K_{*},
$$

where (following the lattice and DT terminology), we set

$$
K_{*}=\frac{d-2}{2 \pi^{2}} \Lambda^{2}\left[3-\frac{\pi}{2}-\log \left(2 \pi^{2}\right)\right],
$$

which is the critical value of the string tension for the given regularization. The physical, renormalized string tension $K$ is then obtained when the bare string tension $K_{0}$ approaches $K_{*}$ from above.

\section{MINIMIZING THE ENERGY AT LARGE $d$}

In the preceding section, the minimization should be performed order by order in the semiclassical expansion, i.e., the small $\alpha^{\prime}$ or large $K_{0}$ expansion. However, there exists another expansion, the large- $d$ expansion [5], where the path integral is expanded around a saddle point and where an infinite set of $\alpha^{\prime}$ corrections is included even to the leading order.

Substituting (11) in Eq. (10) with $a=\pi / \Lambda$, we obtain

$$
\begin{gathered}
S_{0}+S_{1}=\frac{K_{0}}{2}\left(\omega_{R}+\frac{R^{2}}{\omega_{R}}\right) T \\
+\frac{d \Lambda^{2} T}{2 \pi^{2}}\left\{R\left[\log \left(\left(\frac{\omega_{R}}{R}+\frac{R}{\omega_{R}}\right) \pi^{2}\right)-3\right]\right. \\
+\frac{1}{2}\left(\omega_{R}+3 \frac{R^{2}}{\omega_{R}}\right) \arctan \frac{\omega_{R}}{R}+\frac{1}{2}\left(3 \omega_{R}+\frac{R^{2}}{\omega_{R}}\right) \arctan \frac{R}{\omega_{R}} \\
\left.-\frac{\pi}{4}\left(\omega_{R}+\frac{R^{2}}{\omega_{R}}\right)\right\}-\frac{\pi d T}{6 \omega_{R}}
\end{gathered}
$$

for $\omega_{\mathrm{T}}=T \gg R$. A saddle point is reached when $\omega_{R}$ satisfies the equation

$$
\begin{gathered}
\left(\omega_{R}^{2}-R^{2}\right) K_{0}+\frac{d \pi}{3}+\frac{d \Lambda^{2}}{2 \pi^{2}}\left[\left(\omega_{R}^{2}-3 R^{2}\right) \arctan \frac{\omega_{R}}{R}\right. \\
\left.+\left(3 \omega_{R}^{2}-R^{2}\right) \arctan \frac{R}{\omega_{R}}-\frac{\pi}{2}\left(\omega_{R}^{2}-R^{2}\right)\right]=0 .
\end{gathered}
$$

The usual Alvarez-Arvis behavior

$$
E=K_{0} \omega_{R}, \quad \omega_{R}=\sqrt{R^{2}-\frac{\pi d}{3 K_{0}}}
$$

is reproduced from Eqs. (20) and (21) for $\Lambda=0$.

\subsection{Large $R$}

Equation (21) can be solved in the limit of long strings (i.e., large $R$ ), leading to

$$
\begin{gathered}
\omega_{R}=R-\frac{\pi d}{6 R} \frac{1}{\left[K_{0}+d \Lambda^{2}(\pi-2) / 4 \pi^{2}\right]} \\
-\frac{\pi^{2} d^{2}}{72 R^{3}} \frac{1}{\left[K_{0}+d \Lambda^{2}(\pi-2) / 4 \pi^{2}\right]^{2}} \\
-\frac{\pi^{3} d^{3}\left[K_{0}+d \Lambda^{2}(3 \pi-4) / 12 \pi^{2}\right]}{432\left[K_{0}+d \Lambda^{2}(\pi-2) / 4 \pi^{2}\right]^{4} R^{5}}+O\left(R^{-7}\right)
\end{gathered}
$$

and 


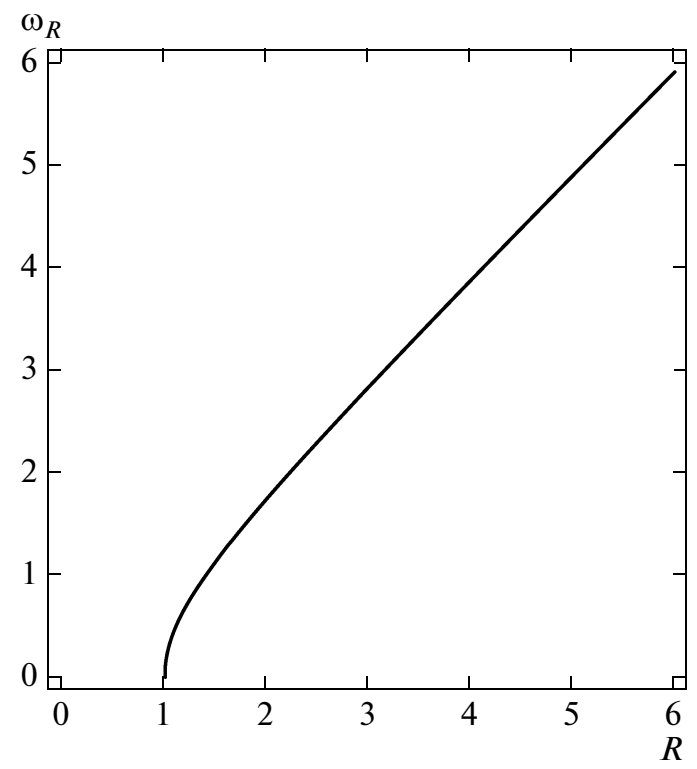

Fig. 1. Plot of the solution of Eq. (21): $\omega_{R}$ versus $R$ in the units of $1 / \Lambda$ for positive $K_{0}$.

$$
\begin{gathered}
E(R)=\left\{K_{0}+\frac{d \Lambda^{2}}{2 \pi^{2}}\left[\log \left(2 \pi^{2}\right)+\frac{\pi}{2}-3\right]\right\} R \\
-\frac{\pi d}{6 R}-\frac{\pi^{2} d^{2}}{72\left[K_{0}+d \Lambda^{2}\left((\pi-2) / 4 \pi^{2}\right)\right] R^{3}} \\
-\frac{\pi^{3} d^{3}}{432\left[K_{0}+d \Lambda^{2}(\pi-2) / 4 \pi^{2}\right]^{2} R^{5}} \\
-\frac{\pi^{4} d^{4}\left[5 K_{0}+d \Lambda^{2}(15 \pi-28) / 12 \pi^{2}\right]}{10368\left[K_{0}+d \Lambda^{2}(\pi-2) / 4 \pi^{2}\right]^{4} R^{7}}+O\left(R^{-9}\right) .
\end{gathered}
$$

In both expansions, we keep the terms that show a deviation from the expansion of the standard squareroot expression (22).

It is seen from Eq. (24) that we cannot simultaneously renormalize all orders of the $1 / R$ expansion. If the string tension is renormalized according to Eqs. (18) and (19), ${ }^{3}$ the dimensional coefficients at the next orders (that are related to the mass of the lowest state) remain $\Lambda$-dependent. This shows some similarity to DT, except that insisting on the mass renormalization there leads to a string tension that cannot be renormalized (it becomes infinite as the cutoff is removed).

\subsection{Small $R$}

For small $R$ (short strings), Eq. (21) can also be analyzed.

\footnotetext{
${ }^{3}$ The string tension is the same as at one loop because the large- $R$ limit is always semiclassical, because the expansion is in $\alpha^{\prime} / R^{2}$.
}

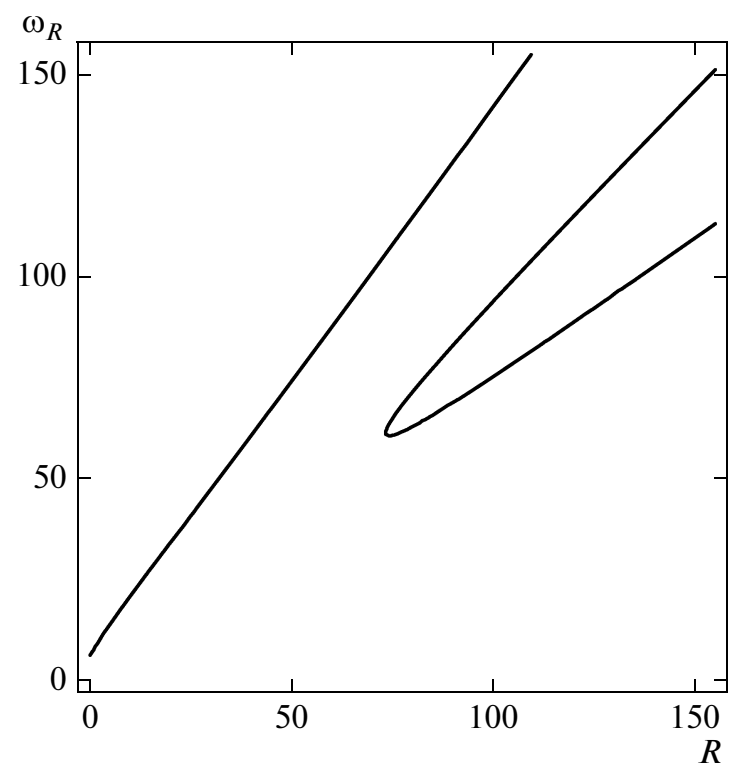

Fig. 2. Plot of the solution of Eq. (21): $\omega_{R}$ versus $R$ in the units of $1 / \Lambda$ for negative $K_{0}>K_{c}$.

For $\Lambda=0$ (the zeta-function regularization), the exact solution (22) shows the appearance of the tachyon, namely, when $\omega_{R}$ ceases to be real for

$$
R<\sqrt{\frac{\pi d}{3 K}}
$$

If we simply set $R=0$ in Eq. (21), the coefficient in front of $\Lambda^{2}$ would vanish and the situation would be like that for the zeta-function regularization.

However, for the distances $R \sim 1 / \Lambda$ and for $\left|K_{0}\right| \sim$ $\Lambda^{2}$, we have $R \sqrt{\left|K_{0}\right| / d} \sim 1$, and hence all terms in Eq. (21) are of the order of unity and therefore remain important for such small $R$. Nevertheless, the equation can be numerically solved for both positive and negative values of $K_{0}$.

In Fig. 1, we plot the solution for $\omega_{R}$ versus $R$ obtained in the units of $1 / \Lambda$ for $K_{0}=d \Lambda^{2}$ by using Mathematica. The solution looks similar to that in Fig. 1 for other positive values $K_{0} \sim d \Lambda^{2}$.

We see from Fig. 1 that the solution looks qualitatively similar to that in Eq. (22). The solution no longer exists for small values of $R \sim 1 / \Lambda$, as already mentioned. We may assume that this is associated with a tachyon of the mass $\sim \Lambda$.

For negative values of $K_{0}$, the situation is different. We see from expansion (23) that it no longer applies for $K_{0}$ near the critical value

$$
K_{c}=-\frac{d \Lambda^{2}(\pi-2)}{4 \pi^{2}}=-0.0289169 d \Lambda^{2} .
$$

The solution for $\omega_{R}$ versus $R$ is plotted in Fig. 2 in the units of $1 / \Lambda$ for $K_{0}=-0.028 d \Lambda^{2}$. It looks similar for 


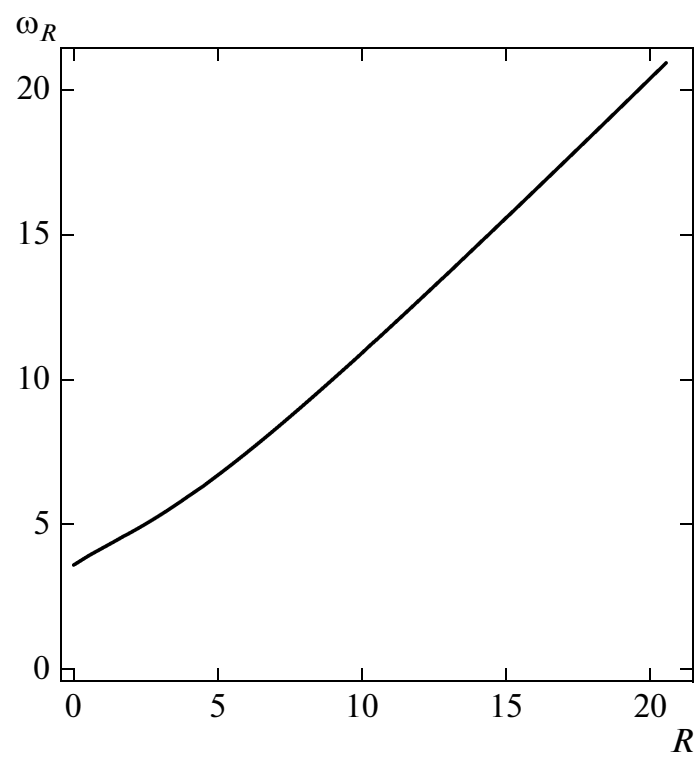

Fig. 3. Plot of the solution of Eq. (21): $\omega_{R}$ versus $R$ in the units of $1 / \Lambda$ for negative $K_{0}<K_{c}$.

other negative $K_{0}>K_{c}$. It can be seen from the figure that a new branch emerges that starts from

$$
\omega_{R}=\sqrt{-\frac{\pi d}{3 K_{0}}},
$$

as is prescribed by the exact formula for the solution of Eq. (21) at $R=0$. This branch emerges whenever $K_{0}$ is negative. For the old branch, which corresponds to expansion (23), the tachyonic instability at $R \sim 1 / \Lambda$ exists as it does for positive values of $K_{0}$. However, this instability goes to infinity (in the units of $1 / \Lambda$ ) as $K_{0}$ approaches critical value (26) from above. This may imply that the tachyon mass scales as $K_{0} \longrightarrow K_{c}+0$.

This old branch disappears for $K_{0}<K_{c}$, given by Eq. (26). The solution exists for all $R \geq 0$. At large $R$, it is given by expansion (23), but now $\omega_{R}>R$ instead of $\omega_{R}<R$, as it was for $K_{0}>K_{c}$. The dependence of the solution of Eq. (21) for $\omega_{R}$ on $R$ is plotted in Fig. 3 for $K_{0}$ near

$$
\begin{aligned}
K_{*}= & \frac{d \Lambda^{2}\left[3-\pi / 2-\log \left(2 \pi^{2}\right)\right]}{2 \pi^{2}} \\
= & -0.0786963 d \Lambda^{2},
\end{aligned}
$$

the value for which the right-hand side of Eq. (18) vanishes. Figure 3 is drawn quantitatively for $K_{0}=K_{*}$. This is the value for which $K$ remains finite as $\Lambda \longrightarrow \infty$, as is discussed below. The solution for $\omega_{R}$ in terms of $\Lambda$ looks similar to that in Fig. 3 for other negative values $K_{0}<K_{c}$.

We see from the figure that the solution always exists, while $\omega_{R} \approx 1 / \Lambda$ for $R=0$, as is prescribed by the exact formula (27) for the solution of Eq. (21) at $R=$ 0 . We may assume that this situation corresponds to the case where there is no tachyon in the spectrum, like for DT.

If $K_{0}$ approaches $K_{c}$ from below, $K_{0} \longrightarrow K_{c}-0$, then the lowest mass is apparently not tachyonic and scales. In this limit, the "renormalized" string tension (18) is a positive constant times $\Lambda^{2}$, since $K_{*}<K_{c}$, and does not scale. It goes to infinity as $\Lambda \longrightarrow \infty$ in complete analogy with the situation for DT in [9]: no tachyon and no scaling of the string tension.

\section{CONCLUSIONS}

We have shown that the regularization of the bosonic string by truncating the mode expansion is feasible and that the spectrum of the regularized string depends on the regularization procedure. In our case $N_{T}=N_{R} T / R$, which maximally preserves the diffeomorphism invariance, the results seem to be somewhat similar to DT for a certain range of the negative bare string tension: the absence of the tachyon and no scaling behavior of the string tension, which remains $\sim \Lambda^{2}$. There exists a (negative) critical value of the bare string tension, for which the mass of the lowest excitation scales as $\Lambda \longrightarrow \infty$, but the renormalized string tension does not. For a certain negative value of the bare string tension, there exists only a simple scaling limit as $\Lambda \longrightarrow \infty$, coinciding with the semiclassical spectrum.

We benefited many times from useful discussions with Valery Rubakov, in particular, on the subject of this paper.

J. A. recalls: I first met Valery when he visited the Niels Bohr Institute for a couple of month in 1984 together with Vadim Kuzmin. We had a wonderful collaboration resulting in an article about hypothetical techni-baryons as nontopological solitons in a technicolor theory. But the most delightful memories from this collaboration are not about physics but about the discussions we had about life and society. We were often working at late night in the basement of the Niels Bohr Institute (we were doing some numerical work and that was where the computer terminals were in these old days). Some time after the midnight, Vadim would come down to us and say: "time to stop working, young guys," and he would bring three glasses and a bottle of vodka, and we would discuss all kind of topics, some of them quite surprizing for Valery. He understood soon that he had been somewhat misinformed about how a society like the Danish one was structured. In particular, I remember a long discussion we had about the Danish health care system. Valery continued asking and asking and in the end he said: "I see, I understand now that it can be organized that way, and be equally good for both the poor and the rich" (which is perhaps not entirely true ...). The openness with which he received the new information and the way he corrected his view after critically judging on whether the information was reliable impressed me immensely. If only people in general could judge 
new cultures the way he did we would live in a better world.

Y. M. recalls: I intersected once with Valery at Fermilab in 1989, where he was giving an honorable colloquium (a week after the one given by Steven Hawking). Before his colloquium, we played volleyball with other members of the Theory Department, including the head, Bill Bardeen. At some point, Bill said to Valery that he should probably go now to a special lunch with some important people in connection with his colloquium. "No," replied Valery, "I have canceled it because of the volleyball game." Our team led by Bill finally lost to the team led by Valery. Bill then formulated: "Whatever Valery does, he does it well."

Our best wishes for the jubilee!

\section{ACKNOWLEDGMENTS}

The authors acknowledge support by the ERCAdvance grant 291092, "Exploring the Quantum Universe" (EQU). J. A. acknowledges support of FNU, the Free Danish Research Council, from the grant "Quantum Gravity and the Role of Black Holes."
Y. M. thanks the Theoretical Particle Physics and Cosmology group at the Niels Bohr Institute for the hospitality.

\section{REFERENCES}

1. A. M. Polyakov, Phys. Lett. B 103, 207 (1981).

2. O. Alvarez, Nucl. Phys. B 216, 125 (1983).

3. B. Durhuus, P. Olesen, and J. L. Petersen, Nucl. Phys. B 198, 157 (1982).

4. J. Ambjorn, Y. Makeenko, and A. Sedrakyan, Phys. Rev. D: Part., Fields, Gravitation, Cosmol. 89, 106010 (2014). arXiv:1403.0893 [hep-th].

5. O. Alvarez, Phys. Rev. D: Part., Fields 24, 440 (1981).

6. J. F. Arvis, Phys. Lett. B 127, 106 (1983).

7. L. Brink and H. B. Nielsen, Phys. Lett. B 45, 332 (1973).

8. J. Ambjørn, B. Durhuus, and J. Fröhlich, Nucl. Phys. B 257, 433 (1985); F. David, Nucl. Phys. B 257, 45 (1985); V. A. Kazakov, A. A. Migdal, and I. K. Kostov, Phys. Lett. B 157, 295 (1985).

9. J. Ambjørn and B. Durhuus, Phys. Lett. B 188, 253 (1987). 